ISSN: 2600-5859

\title{
Perfeccionamiento de la enseñanza de la estadística en la carrera de turismo (c) (1)(2)
}

Improvement of the teaching of statistics in the tourism career

MSc. Elena Fraga Guerra. ${ }^{1} \&$ Dr. Roberto Llanes Pérez. ${ }^{2}$

Recibido: 09-12-2019 / Revisado: 12-12-2019 / Aceptado: 16-12-2019 / Publicado: 03-01-2020

Resumen.

DOI: https://doi.org/10.33262/concienciadigital.v3i1.1005

Uno de los retos fundamentales que enfrenta hoy en día la educación superior cubana es el diseño e implementación de una nueva generación de planes de estudio de las carreras universitarias, denominados Planes de Estudio E. En este trabajo se exponen consideraciones acerca de los retos que implica el perfeccionamiento del proceso de enseñanza - aprendizaje de la estadística en la carrera de Turismo en este nuevo contexto, y a partir de ello se realizan propuestas para el perfeccionamiento de la enseñanza de la asignatura, tanto para el curso diurno como para el curso por encuentros.

Palabras claves: retos fundamentales, planes de estudio, educación superior cubana.

\section{Abstract.}

One of the fundamental challenges facing Cuban higher education today is the design and implementation of a new generation of curricula for university careers, called Study Plans E. In this work, considerations are made about the challenges involved in perfecting the teaching process - learning statistics in the Tourism career in this new context, and based on this, proposals are made for perfecting the teaching of the subject, both for the daytime course and for the encounter course.

\footnotetext{
${ }^{1}$ Dirección de Formación de Profesionales, MES, Cuba, efraga@mes.gob.cu

${ }^{2}$ Facultad de Turismo, Universidad de la Habana, Cuba, roberto.llanes@ftur.uh.cu
} 
Keywords: fundamental challenges, curricula, Cuban higher education.

\section{Introducción.}

El objetivo fundamental de la educación superior cubana lo constituye la formación de profesionales integrales con un alto nivel de competitividad, lo que implica un reto para las instituciones de educación superior y otras entidades vinculadas a este proceso. En ello se debe trabajar sistemática y científicamente, lo que presupone la realización de un perfeccionamiento continuo del proceso de enseñanza - aprendizaje.

En la actualidad, la educación superior en nuestro país se encuentra inmersa en la implementación de una nueva generación de planes de estudio, denominada Planes de Estudio E. En esta nueva generación de planes de estudio, la enseñanza de la estadística en la carrera de Turismo ha tenido un proceso de modificaciones sustanciales que imponen al claustro de la Facultad retos en la enseñanza de esta ciencia en un nuevo contexto.

Las bases conceptuales de los Planes de Estudio E se fundamentan en la formación de profesionales de perfil amplio, capaces de resolver los problemas más generales y frecuentes del eslabón de base de la profesión y preparados para asumir su formación continua. El tiempo de horas presenciales se reduce con respecto a planes de estudio anteriores y se fomenta un mayor protagonismo y autonomía de los estudiantes durante sus estudios universitarios mediante el empleo de las tecnologías de la información y las comunicaciones.

Por otra parte, se hace necesario reforzar la motivación de los estudiantes por el estudio de la estadística, así como potenciar los métodos de estudio para una mejor asimilación de los contenidos, métodos de enseñanza que propicien una mejor comprensión e interpretación de los modelos y métodos estadísticos, la resolución de problemas vinculados con el perfil del egresado, así como una mejor preparación para asimilar contenidos posteriores en la carrera que tienen como base conceptual la estadística y la realización de trabajos investigativos que incluyen el ejercicio de culminación de los estudios. Es imprescindible que los estudiantes de la carrera de Turismo conozcan y apliquen los métodos estadísticos que le permitan organizar, sistematizar, procesar y analizar la información relacionada con las actividades turísticas para la toma de decisiones.

Para este análisis se aplicaron métodos tales como el análisis documental, la observación en clases, los resultados de las evaluaciones realizadas a los estudiantes, sus criterios sobre los contenidos impartidos, así como el desempeño de estos en otras asignaturas y en la realización de los ejercicios de culminación de los estudios de la carrera en los que es necesario la aplicación de estos métodos estadísticos. 
En este trabajo se exponen consideraciones de los autores, quienes poseen una vasta experiencia en la enseñanza de la estadística en la educación superior, acerca de los retos que implica el perfeccionamiento del proceso de enseñanza - aprendizaje de esta ciencia en la actualidad. A partir de ello se realizan propuestas para el perfeccionamiento de la enseñanza de la asignatura, tanto en el curso diurno como en el curso por encuentros, en la carrera de Turismo.

Las propuestas están sustentadas en la articulación de la asignatura con el modelo del profesional, la adecuada orientación del estudio independiente que incluya la incorporación de preguntas que le permita al estudiante profundizar y aplicar métodos adecuados de estudio, entre otros aspectos, la elaboración de materiales de apoyo a la enseñanza, la introducción de métodos activos de enseñanza y el perfeccionamiento del sistema de evaluación.

\section{Desarrollo.}

\section{Retos ante una nueva generación de planes de estudio en las universidades cubanas.}

La educación superior tiene como misión: preservar, desarrollar y promover la cultura de la humanidad, a través de sus procesos sustantivos (formación, investigación y extensión) y en estrecho vínculo con la sociedad; contribuyendo a su desarrollo sostenible.

Por ello las universidades deben ser pertinentes, no solo en el sentido de reproducción y legitimación de la vida económica, social y cultural, sino también en el sentido crítico, renovador y transformador, altamente comprometida con el desarrollo cultural, económico y social del país, dándole amplia respuesta a las necesidades de la educación postgraduada y que la investigación científica sea un componente consustancial del quehacer universitario.

La formación de los futuros profesionales hoy en día se encamina a potenciar la asimilación de nuevos contenidos, desarrollar la capacidad creativa y de innovación, de incorporar la metodología científica en la investigación y las diversas técnicas y métodos para el estudio y análisis de resultados y que los egresados mantengan y fortalezcan los hábitos de una educación permanente, de una formación continua, entre otros aspectos, sin olvidar la formación de valores en los estudiantes, durante el proceso académico, con el fin de lograr un profesional comprometido con el país y su proyección social.

¿Cómo entonces las universidades garantizan la formación de un egresado integral?

En el diseño de los planes de estudio de una carrera universitaria, en indispensable que se tengan en cuenta las exigencias de la época, las exigencias propias del país, de la región, de su sistema social y las exigencias específicas de la profesión, con una visión que posibilite al egresado dar soluciones a problemas que se le pueden presentar posteriormente en su vida laboral.

$\mathrm{Al}$ graduado universitario se le presentan en su labor profesional exigencias propias de sus funciones y que devienen en los objetivos del egresado, a saber: 
1. La apropiación de nuevos conocimientos y habilidades, que implica la continua superación para mantenerse actualizado en el campo de su especialidad.

2. La transmisión de conocimientos científicos.

3. La aplicación de los conocimientos científicos.

4. La actividad creadora en el desarrollo de los conocimientos científicos, que incluye la realización de trabajos de investigación, la búsqueda de nuevas soluciones, métodos y procedimientos en la ciencia y la tecnología.

En los nuevos planes de estudio E, implementados en la educación superior cubana, el concepto de formación básica es asumido con mayor amplitud. El currículo se divide en: currículo base (son los contenidos precisados por las Comisiones Nacionales de Carrera y de obligatorio cumplimiento para todos las universidades), currículo propio (son los contenidos, precisados por cada universidad, en correspondencia con las particularidades del proceso de formación en cada carrera y centro) y el currículo optativo/electivo (son los contenidos que son escogidos por cada estudiante que sirven de complemento para su formación integral), las asignaturas y disciplinas deben evidenciar un mayor nivel de esencialidad, debe haber una mejor correspondencia entre la profesión y las ciencias que a ella tributan.

Por otra parte, se sustentan en la disminución de la carga semanal de horas presenciales, las transformaciones relacionadas con la virtualización del proceso de formación, una formación humanística más completa y el establecimiento de sistemas de evaluación del aprendizaje cualitativos e integradores, centrados en el desempeño de los estudiantes, por mencionar algunos; lo que supone cambios en el modo de actuar y de pensar de todos los implicados en este proceso.

También se deben tener en cuenta la implementación de las estrategias curriculares, como son el uso de las Tecnologías de la Información y las Comunicaciones, el conocimiento del idioma inglés, la formación económica, las técnicas de dirección, el empleo correcto de la información científico técnica y la dimensión medio ambiental, entre otras.

Ello supone que las prioridades sean:

1.La aplicación del enfoque integral, con énfasis en la labor educativa desde el contenido de las disciplinas.

2.El desarrollo de habilidades en los estudiantes que les posibilite un pensamiento creativo, la independencia, el análisis para la toma de decisiones.

3. La actividad investigativo-laboral, con un enfoque más abierto, que implique, en los casos que lo requieran, la formación desde el trabajo.

4. Consolidación del sistema de evaluación. 


\section{Rol de las matemáticas en la carrera de Turismo.}

La matemática como ciencia, contribuye al desarrollo de los pensamientos abstracto, lógico y algorítmico, y aporta los fundamentos básicos para un egresado de la carrera de Turismo, dado que este profesional requiere modelar en términos matemáticos, su objeto de trabajo: "conjunto de fenómenos y relaciones económicas, psico-sociológicas, culturales y medioambientales que se generan en los procesos asociados a las actividades turísticas, entre los visitantes temporales, las entidades vinculadas a los viajes y las comunidades de acogida con motivo de sus viajes y estancias en lugares diferentes a los de su residencia habitual" (4), así como en los procesos afines, con los cuales refleja los rasgos cuantitativos y cualitativos de los fenómenos que estudia.

Por consiguiente, estos futuros profesionales deben poseer una adecuada preparación matemática y estadística que comprende, además de los conocimientos teóricos, el correspondiente a los programas computacionales: matemáticos, estadísticos, de gestión y otros, que comprenden una correcta interpretación de los resultados. Esta disciplina contribuye a:

- Ampliar la madurez matemática y la capacidad de trabajo con la abstracción.

- Desarrollar habilidades para la comunicación y comprensión de propiedades y características matemáticas de magnitudes y formas en las variantes formal, gráfica, numérica y verbal.

- La conformación de una cultura científica general e integral actualizada.

- Identificar, interpretar y analizar modelos matemáticos de diversos procesos vinculados a la profesión, así como resolver los problemas de índole matemática a los que éstos conducen, utilizando para ello los contenidos matemáticos que se estudian en la disciplina, haciendo un uso eficiente de las Tecnologías de la Información y las Comunicaciones.

- Construir una sólida base de conocimientos, integrada y sistémica, que deje huella en su proceso de aprendizaje y le permita resolver problemas con los recursos y estrategias estudiadas.

- Aprenda a pensar y actuar de forma creadora.

Para ello se requiere una concepción del modelo de enseñanza que tenga en cuenta:

1. Una estructuración sistémica de los contenidos (conocimientos, habilidades y valores).

2. Una enseñanza centrada en el estudiante como sujeto activo, constructor y reconstructor de su propio conocimiento y proceso de aprendizaje.

3. Una enseñanza a través y para la resolución de problemas vinculados a la carrera y a otras disciplinas y asignaturas.

4. Una enseñanza desarrolladora dirigida a la educación de la personalidad del estudiante con una implicación personal activa, consciente y reflexiva que le provoque 
satisfacción, con la necesaria flexibilidad, independencia, perseverancia, y una actitud ante la vida responsable y autodeterminada que se proyecte con una perspectiva temporal mediata.

En el actual Plan de Estudios E de la carrera de Turismo está presente la disciplina Matemática Aplicada e Informática, cuyo contenido básico es la recolección, organización y tratamiento de los datos, su tabulación y representación gráfica, el uso de técnicas matemáticas y estadísticas para el estudio de las relaciones cuantitativas en los procesos y fenómenos del turismo, el uso de algunos de los modernos medios y técnicas para el procesamiento y presentación de la información, así como de las nuevas tecnologías del comercio electrónico empleadas en el turismo, posibilitando el cuerpo teórico e instrumental necesario en una primera fase de la base curricular de estudio. (4)

Las asignaturas que conforman esta disciplina contribuyen a la formación básica de conocimientos y habilidades propios y de base instrumental para el desarrollo de otras disciplinas y sus asignaturas, y tienen como sistema de conocimientos: Funciones y ecuaciones. Derivadas y análisis marginal. Integrales. Matrices y sistemas de ecuaciones lineales. Programación lineal. Probabilidades. Estadística descriptiva e inferencial. Matemática financiera. Organización y procesamiento de la información. Uso de redes e internet. Software básico y de gestión. (4)

\section{Análisis de la asignatura Estadística aplicada al Turismo.}

La Estadística es una de las ramas de la Matemática que se incluye dentro de la disciplina Matemática Aplicada e Informática, siendo el nombre de la asignatura Estadística aplicada al Turismo, que se imparte en el segundo semestre del primer año del Curso Diurno y en el primer semestre del segundo año en el Curso por Encuentros.

La propia naturaleza de la actividad a desarrollar por cualquier profesional del turismo hace que la Estadística sea una herramienta fundamental en las actividades propias de carácter operativo y en los estudios e investigaciones de análisis, estratégicos y de planificación. En el caso del turismo, la estadística permite y facilita, entre otras acciones:

1. Estudiar y sistematizar la evolución de la realidad turística, la estacionalidad, el crecimiento, los ciclos, los impactos de la actividad, etc., así como pronosticar comportamientos de dicha realidad.

2. Conocer la evolución de un determinado destino turístico valorando su gestión a corto y largo plazo.

3. Entender los eventos que se producen dentro del sector, proporcionando información útil a los decisores y planificadores de políticas y estrategias.

4. Reducir el tiempo de incertidumbre y el tiempo de reacción ante los cambios en los requerimientos de operadores turísticos, proveedores, clientes y del medio ambiente.

5. Analizar los errores y logros observados en el pasado con el fin de establecer políticas correctoras o potenciar las estrategias de futuro. 
6. Explicar y realizar informes sobre el control de los procesos, estableciendo predicciones sobre escenarios futuros que serán la base de la toma de decisiones.

7. Facilitar a las empresas turísticas, el adaptarse a los nuevos mecanismos de mercado y a los procesos de modernización impuestos por las nuevas tecnologías, permitiendo aumentar la competitividad al permitir un mayor conocimiento del mercado y del entorno en el que se desarrolla la actividad turística, proporcionándole un valor añadido que la diferencie del resto de sus competidoras.

8. Suministrar información sobre aspectos como: movimiento de turistas, el perfil del turista, motivaciones de elección de un destino turístico, hábitos de consumo y de compra, satisfacción del consumidor, etc.

El conjunto de contenidos que le precede a la asignatura hace énfasis en el estudio de modelos matemáticos referidos a los fenómenos determinísticos y es en la asignatura Estadística Aplicada al Turismo donde se enfrentan por primera vez al estudio y modelación matemática de los fenómenos aleatorios, lo que constituye un cambio sustancial en la forma del pensamiento de los estudiantes.

Los conocimientos esenciales para adquirir dentro de la asignatura son: Breve historia de la Estadística. La Investigación Estadística. La formación y utilidad del conocimiento estadístico. Recolección, organización y representación de los datos. Distribuciones de frecuencias unidimensionales y bidimensionales. Correlación y Regresión Lineal simple. Series Temporales. Fenómenos deterministas y fenómenos aleatorios. Probabilidades y distribuciones probabilísticas. Distribución normal. Muestreo. Estimación puntual y por intervalos de confianza. Elementos conceptuales de prueba de hipótesis y análisis de varianza. Diseño y tratamiento estadístico de encuestas. El uso de los paquetes de programas estadísticos para el procesamiento de los datos.

Su sistema de evaluación en el anterior Plan C se sustentaba en la realización de 2 trabajos extra-clase: el primero sobre la aplicación de los conocimientos y habilidades impartidas hasta el tema de Regresión Lineal y el segundo sobre Series Cronológicas comprendiendo el Análisis de la Evolución, Estacionalidad y Pronóstico de los arribos turísticos desde un mercado emisor a Cuba. Además, se finalizaba con una prueba parcial sobre la Inferencia Estadística.

En la información enviada para la formulación del Plan E, al referenciar la Evaluación Frecuente (EF), se entendió Evaluación Final fijándose así en el Plan E, modificándose totalmente el Sistema de Evaluación de la Asignatura, el cual comprende evaluaciones parciales y el examen final.

En la enseñanza de la asignatura, a lo largo de varios años, se muestra la solidez del claustro de profesores que la imparten y avances en el trabajo científico-metodológico, la elaboración de numerosos materiales de apoyo a la docencia y el texto de la asignatura en su etapa final de elaboración, el montaje de la asignatura en la plataforma Moodle, la participación del 
claustro como tutores, oponentes o como miembros de tribunal en Trabajos de Diploma, Tesis de Maestría y Tesis de Doctorado o en actividades de carácter científico - estudiantil, la orientación de tareas extra-clases donde el estudiante debe resolver problemas vinculados al ejercicio de la profesión y para los cuales deben aplicar métodos estadísticos, entre otros. Sin embargo, aún se evidencias insuficiencias en la adquisición de conocimientos, habilidades y valores por parte de los estudiantes a los cuales debe tributar la asignatura, y que se relacionan a continuación:

1. Es insuficiente el dominio de contenidos relacionados con el trabajo algebraico, las representaciones gráficas, y otros que se imparten en la enseñanza precedente, no mostrándose habilidades en el proceso de razonamiento o análisis de las situaciones o problemas a modelar o plantear, lo que dificulta el aprendizaje de la asignatura.

2. No son pocas las veces que los estudiantes no se sienten motivados por el estudio de la asignatura por su nivel de complejidad e incomprensión de la utilidad de la misma y muchos por rechazo a las situaciones cuantitativas que requieren dominio de las técnicas correspondientes, no asimilando correctamente los conceptos y técnicas aplicadas.

3. En general, existe una tendencia por parte de los estudiantes a resolver los problemas propuestos mecánicamente, sin un análisis previo de los contenidos teóricos de la asignatura, por lo que no interiorizan correctamente los pasos a seguir y tampoco interpretan correctamente los resultados. Es posible que sean capaces de memorizar fórmulas y los pasos a seguir en problemas, pero solo raramente parecen tener sentido en lo racional o en como los conceptos se aplican a nuevas situaciones.

4. Es insuficiente aún la orientación para la búsqueda de información referente a la aplicación de los métodos estadísticos a las investigaciones y soluciones de problemas vinculados con su especialidad y del desarrollo de la Estadística en cuanto al espacio cognoscitivo y su importancia en el quehacer investigativo.

5. Por otra parte, en el plan de estudios de la carrera, existen asignaturas cuya comprensión y por ende su aprendizaje, precisan de sólidos conocimientos de estadística; y no son pocas las veces que se evidencian dificultades en ello.

\section{Propuesta.}

Con el objetivo de perfeccionar el proceso de enseñanza - aprendizaje de la asignatura, sea hace imprescindible profundizar en su vinculación con el modelo del profesional y en su articulación horizontal y vertical con otras asignaturas de la carrera en el contexto actual de los planes de estudio E; no solo en lo referente al sistema de contenidos, si no en las propias bases conceptuales de estos planes de estudios que apuestan por la formación de un profesional preparado para ejercer en el eslabón de base de la profesión y con una mayor independencia durante el proceso de formación. 
Uno de los aspectos que se debe tener en cuenta en la enseñanza de la asignatura, es la dedicación, en las actividades lectivas, de un mayor espacio en el tema introductorio sobre la historia de la estadística, la investigación estadística y la formación y utilidad del conocimiento estadístico pues les permite conocer las cuestiones que dieron lugar a los diversos conceptos, el origen de los términos, lenguajes y notaciones, los problemas que se han ido resolviendo, y su evolución hasta la actualidad. Hacer comprender a los estudiantes que el conocimiento matemático no es algo totalmente acabado sino en plena creación y su vital importancia en el desarrollo intelectual e investigativo.

Se debe potenciar aún más en las actividades docentes la formación de habilidades de modelación, algoritmización, representaciones gráficas, habilidades de cálculo e interpretación de resultados, lo que tiene una incidencia directa en la capacidad de generalizar métodos de trabajo propios de la asignatura y por ende, en la posibilidad de que el estudiante sea capaz de resolver problemas similares en los años superiores de la carrera y en su labor profesional. No se debe olvidar que se pretende ante todo que el estudiante asuma una posición protagónica en su proceso formativo.

Igualmente es importante, que previo a la impartición de un nuevo contenido (conferencias) según la secuencias de actividades de la asignatura, se oriente el estudio de determinados aspectos relacionados con el sistema de contenidos de la conferencia, y que a partir de este estudio los estudiantes identifiquen las ideas claves, elaboren esquemas de llaves, esquemas numéricos y esquemas procedimentales y/o realicen un resumen de los aspectos más significativos, según el caso, lo que posibilita la aplicación de los métodos activos de enseñanza.

Es esencial mostrar que los contenidos que se desarrollan en esta asignatura contribuyen a resolver problemas reales de la especialidad, lo que posibilita que de una manera consciente los estudiantes participen de forma activa en la solución de problemas vinculados al ejercicio de la profesión; por lo que en el desarrollo de las clases se plantea la necesidad de fortalecer la inclusión de ejemplos de casos reales en los cuales sean aplicados los métodos estadísticos que correspondan, sin dejar de prestar atención en la realización de ejercicios propios de la asignatura y no centrar solo su enseñanza en el carácter utilitario en la solución de problemas de la especialidad.

En el caso de los cursos por encuentros hay una mayor potencialidad para plantear y resolver problemas propios de los estudiantes relacionados con los contenidos de los trabajos o responsabilidades según sus funciones. En el curso diurno estos problemas pueden serles planteados como casos de estudio.

Se debe potenciar la autonomía en el aprendizaje, lo que presupone el reto de que más que enseñar contenidos se debe facilitar que los alumnos desarrollen habilidades, actitudes y valores que proyecten un aprendizaje efectivo en el saber. La autonomía proyecta de los sujetos una facultad de tomar decisiones, de participación automotivados en la aplicación de 
los recursos adquiridos por medios de experiencias de aprendizaje, realizadas en el contexto escolar y fuera de él; y que los han capacitado para responder a las necesidades y retos del entorno.

El estudiante tiene que aprender a buscar, seleccionar, analizar críticamente e integrar en sus esquemas cognitivos, la información necesaria para desenvolverse con éxito en la sociedad. Tan importante es aprender determinados contenidos conceptuales fundamentales, como lo es aprender procedimientos y estrategias para manejar la información, que le permitirá continuar aprendiendo a lo largo de la vida.

Debe fortalecerse ampliar los Laboratorios de Computación donde se impartan softwares profesionales que le permiten, a través de la actividad del estudiante, que este se apropie de los conocimientos y las técnicas estadísticas que necesita.

Hoy en día, en la enseñanza de la estadística se hace imprescindible el uso de las tecnologías de la información y las comunicaciones, de hecho constituyen fuentes de búsqueda de información actualizada, que además posibilitan la ampliación de conocimientos de los estudiantes en esta ciencia. Existen además tutoriales y programas de ejercitación que permiten tareas de reconocimiento, comparación y resolución de problemas; programas que posibilitan la simulación de fenómenos y los programas no diseñados inicialmente para la enseñanza, pero que en la práctica se están utilizando para esta finalidad, son: los procesadores de texto, las hojas de cálculo, generadores de gráficos, paquetes estadísticos y bases de datos.

Es importante que el colectivo de profesores diseñe materiales de apoyo a la docencia en el que se refuerce la consolidación de conocimientos de la enseñanza precedente y que son imprescindibles dominar para una mejor comprensión de la asignatura, ya sea a través de aspectos de carácter teórico, ejercicios resueltos y propuestos, así como en actividades autoevaluativas que le permitan al estudiante medir sus conocimientos acumulados en la asignatura.

En el diseño del sistema de evaluación se debe definir previamente: el objetivo de la evaluación, en correspondencia con ello el sistema de contenidos, cómo debe ser la evaluación y en qué momento se realiza, de manera tal que sea integral, sistemática, flexible, participativa y formativa. Puede incluir aspectos teóricos y prácticos vinculados a ejercicios integradores; así como, contenidos de carácter académico, laboral e investigativo. En los cursos por encuentros, las tareas o trabajos a desarrollar.

Pueden incluirse preguntas de retroalimentación del contenido anterior, búsqueda de información en la red (internet e intranet) de artículos relacionados con el desarrollo de la estadística como rama de la matemática y de artículos en los que se le dé solución a un problema relacionado con la especialidad en el que para su solución se necesite del empleo de los métodos estadísticos de investigación. 
Se orienta la realización de dos trabajos de curso tal y como estuvo diseñada la asignatura en el que se plantean dos problemas de investigación asociados a la especialidad en el que sea necesario el uso de los métodos estadísticos, que deben ser defendidos por el estudiante o un pequeño equipo de estudiantes y que en el mismo se exija determinada estructura para la presentación de trabajos, como son la elaboración del resumen, introducción, fundamentación teórica, solución, conclusiones, recomendaciones y la bibliografía empleada. Para ello es posible trabajar en conjunto con los profesores de la disciplina principal integradora. En la realización de este trabajo se puede potenciar el uso de las estrategias curriculares, como son el uso de las TIC, la formación económica, el uso del idioma inglés y el empleo correcto de la información científico - técnica.

\section{Conclusiones.}

- Teniendo en cuenta que los modelos y métodos estadísticos constituyen una herramienta fundamental de apoyo a la toma de decisiones vinculadas a un determinado problema planteado a los especialistas cuando son empleados adecuadamente, es importante que la formación estadística que reciben los futuros profesionales del Turismo sea amplia y requiere además de una mayor articulación con las disciplinas del plan de estudio que reciben los estudiantes.

- La aplicación correcta de los métodos estadísticos para las investigaciones ayuda considerablemente a la marcha del proceso docente, desarrollando en el estudiante el hábito de utilizar el método científico de trabajo como herramienta para el enfoque y solución de problemas.

- En general, es importante resaltar, que dada la estrecha vinculación de las mismas con los problemas propios de la especialidad, no se debe olvidar la realización de ejercicios, tanto en clases como en las evaluaciones, que tengan en cuenta el objeto de trabajo de la profesión, los campos de acción y los modos de actuación, para que el estudiante se encuentre en una situación similar a la que se le puede presentar en su vida profesional y tomar sus propias decisiones. Es obvio que en los cursos por encuentros esta posibilidad es mayor dadas las ocupaciones de los estudiantes en sus respectivas empresas.

- Debe ser diseñada de tal forma que le permita al profesor indagar sobre el grado de aprendizaje y desarrollo de los estudiantes en su proceso de formación, así como la capacidad que poseen para aplicar los contenidos en la resolución de problemas de la profesión. 


\section{Referencias bibliográficas.}

Batanero, C. (2001). Didáctica de la estadística. Granada: Grupo de Investigación en Educación Estadística (disponible en http://www.ugr.es/local/batanero).

Colectivo de autores, (2003) Preparación pedagógica integral para profesores universitarios, Editorial Félix Varela, Cuba.

Horruitiner Silva Pedro (2006); La universidad cubana: el modelo de formación, Editorial Félix Varela, Cuba.

Ministerio de Educación Superior, (2017) Plan de Estudio de la Carrera de Turismo.Montiel Torres Ana y col (2007), Estadística para turismo. Primera edición, Editorial McGraw-Hill/Interamericana de España, S. A. U.

Salinas, Jesús (2004). "Innovación docente y uso de las TIC en la enseñanza universitaria". Revista de Universidad y Sociedad del Conocimiento (RUSC).

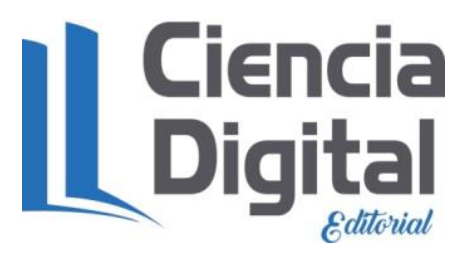


ISSN: 2600-5859

Para citar el artículo indexado.

Fraga Guerra, E., \& Llanes Pérez, R. (2020). Perfeccionamiento de la enseñanza de la estadística en la carrera de turismo. ConcienciaDigital, 3(1), 50-62. https://doi.org/10.33262/concienciadigital.v3i1.1005

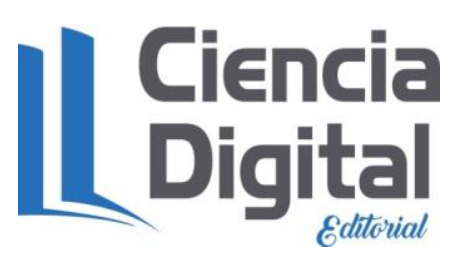

El artículo que se publica es de exclusiva responsabilidad de los autores y no necesariamente reflejan el pensamiento de la Revista Conciencia Digital.

El articulo queda en propiedad de la revista y, por tanto, su publicación parcial y/o total en otro medio tiene que ser autorizado por el director de la Revista Conciencia Digital.
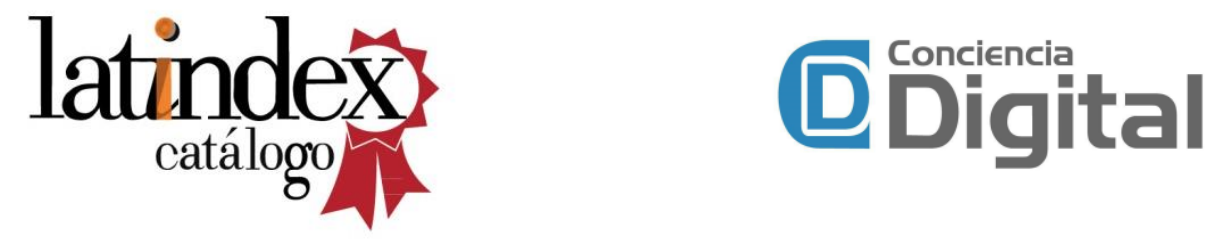